\title{
Discrete Crouzeix-Velte decomposition for the disk domain
}

\author{
Gy. Strauber
}




\title{
DISCRETE CROUZEIX-VELTE DECOMPOSITION FOR THE DISK DOMAIN
}

\author{
GY. STRAUBER
}

[Received: July 21, 2004]

\begin{abstract}
In this paper, a staggered grid approximation of the Stokes problem on the unit disk is investigated. A full description of the approximation and the discrete Crouzeix-Velte decomposition is given in the case of homogeneous Dirichlet boundary conditions. Further we consider the Uzawa algorithm combined with the conjugate gradient method and FFT algorithm for solving the discrete Poisson equations. Computational results show the effectiveness of this discretization.
\end{abstract}

Mathematics Subject Classification: 65N06, 76D05, 35Q30

Keywords: Stokes problem, Crouzeix-Velte decomposition, unit disk, staggered grid approximation

\section{InTRODUCTION}

The Crouzeix-Velte decomposition is a decomposition into three orthogonal subspaces of the customary Sobolev space $\left(H_{0}^{1}(\Omega)\right)^{d}$ of vector functions defined over a lipschitzian domain $\Omega \subset \mathbb{R}^{d}$ which was first described in [4] and later, independently, in [18]. This decomposition is similar to the well-known Helmholtz-Weyl decomposition but different from the latter; not only by the prescribed boundary conditions (originally homogeneous Dirichlet conditions) but also by the properties of the functions in the three subspaces. E.g., along with the subspaces of divergence-free and rotation-free functions, there is a third subspace the functions of which have both nonzero divergence and rotation or are the zero function. In [18] and [5], the decomposition was used to get more information about the inf-sup constant of the Stokes problem. The optimal inf-sup constant can be characterized by the third subspace alone [12].

For the numerical solution of the Stokes problem and its connection to the inf-sup problem, see [1], [9], [2]. In [3], additional results were gained about the inf-sup constant for ring and disk domains.

If using finite difference methods for approximating the Stokes equations, and if the discrete scheme admits a discrete Crouzeix-Velte decomposition, then the same conclusions as in the continuous case can be drawn. The subspaces of the discrete 
rotation-free and discrete divergence-free functions are in a similar relation as the corresponding subspaces of $\left(H_{0}^{1}\right)^{n}$, and there is a third orthogonal subspace consisting of discrete harmonic functions. The optimal constant of the discrete inf-sup condition can therefore be computed in this much smaller third space. These results are of interest for the iterative solution of stationary problems for the Stokes and linear elasticity equations, and for long-term calculations in the time-dependent case for the following reasons: if there exists a Crouzeix-Velte decomposition, then independently of the domain and its triangulation, there is an eigenvalue 1 of high multiplicity (of the order of inner grid points). Then, the error components lying in the eigensubspace corresponding to this eigenvalue can be removed by one step of a simple damped Jacobi iteration. The remaining error is connected only with boundary effects. Since in the two-dimensional case the spectrum is symmetric with respect to $1 / 2$, the optimal iteration parameters can be calculated using only a lower estimate of the inf-sup constant. Moreover, the conjugate gradient iteration automatically takes advantage of such a spectrum and converges faster than for discrete spaces without a decomposition.

In [13], the Uzawa and Arrow-Hurwitz iterations are investigated and are shown to reach the third Crouzeix-Velte subspace after at most 2 steps, and an improved convergence estimate is derived for the conjugate gradient method using the estimates of the inf-sup constant. In [16], the well-known staggered grid approximation of the Stokes problem is investigated. The existence of the discrete Crouzeix-Velte decomposition is proved in the case of a non-equidistant grid for the Shortley-Weller approximation, for a finite volume scheme and in the case of second order approximation and homogeneous Dirichlet boundary conditions. The first order staggered grid approximation in a special case is investigated in [12]. This result was generalized in [6] to general two and three-dimensional domains. In [14], the Crouzeix-Velte decomposition is generalized with respect to boundary conditions and boundary value problems. Moreover, in the discrete case, the gap between the lower-order staggered grid and finite volume approximations and the high-order Scott-Vogelius elements is filled by proving that Fortin-Soulie elements also admit such a decomposition.

The aim of the present paper is to investigate the staggered grid approximation of the Stokes problem in a polar coordinate system, and to prove the existence of the discrete Crouzeix-Velte decomposition for the unit disk, in the case of second order approximation and homogeneous Dirichlet boundary conditions. Further we present computational results using the Uzawa algorithm (as outer iteration) and solving the discrete Poisson equations by the conjugate gradient method (as inner iteration) with an effective preconditioning matrix and FFT algorithm.

The outline of the paper is as follows. In Section 2, the necessary notations and the Crouzeix-Velte decomposition in the continuous case as well as the Stokes problem in the polar coordinate system are introduced. In Section 3, the discrete Stokes 
problem is described. In Section 4, the discrete Crouzeix-Velte decomposition is investigated, and it is shown that this decomposition exists for the disk. Finally, in Section 5 we show the computational results. The results show that the discretization obeying a discrete Crouzeix-Velte decomposition leads to effectively solvable systems of algebraic equations. The average number of the outer iterations using the Uzawa algorithm was 3-5, and the number of inner iterations needed to reach the stopping criterion of the conjugate gradient method, using the best preconditioning matrix, was only 2.

\section{Notations}

2.1. The Stokes problem on the unit disk. Let $\Omega$ be the unit disk

$$
\Omega=\{(r, \varphi) \mid 0 \leq r<1,0 \leq \varphi<2 \pi\},
$$

and consider the following Stokes problem:

$$
\begin{aligned}
\Delta_{r \varphi} u-\frac{u}{r^{2}}-\frac{2}{r^{2}} \frac{\partial v}{\partial \varphi}-\frac{\partial p}{\partial r} & =f_{1}, \\
\Delta_{r \varphi} v-\frac{v}{r^{2}}+\frac{2}{r^{2}} \frac{\partial u}{\partial \varphi}-\frac{1}{r} \frac{\partial p}{\partial \varphi} & =f_{2}, \\
\operatorname{div} \vec{u}=\frac{1}{r}\left(\frac{\partial}{\partial r}(r u)+\frac{\partial v}{\partial \varphi}\right) & =0,
\end{aligned}
$$

where $(u, v)=\vec{u}$ and $\left(f_{1}, f_{2}\right)=\vec{f}$ and

$$
\Delta_{r \varphi}=\frac{1}{r} \frac{\partial}{\partial r}\left(r \frac{\partial}{\partial r}\right)+\frac{1}{r^{2}} \frac{\partial^{2}}{\partial \varphi^{2}} .
$$

On the boundary, homogeneous Dirichlet boundary conditions are imposed:

$$
\vec{u}=0 \text {, on } \partial \Omega \text {, }
$$

The problem consists in finding a vector-function $\vec{u}(x)$ and a scalar function $p(x)$ that satisfy the system of partial differential equations above. The function $\vec{u}$ is the velocity of fluid and $p$ is the pressure. We mention that the appearance of $\mathrm{v}$ in equation (2.1) and the appearance of $u$ in equation (2.2) cause difficulty during the numerical solution of (2.1)-(2.4).

A unique weak solution $\vec{u} \in V$ and $p \in P$ exists when, for example, $\vec{f} \in\left(L_{2}(\Omega)\right)^{n}$, (see, e.g., [11],[17]), where $P:=L_{2,0}(\Omega)$ the subspace of $L_{2}(\Omega)$ of square integrable functions with zero integral over $\Omega$ and $V:=\left(H_{0}^{1}(\Omega)\right)^{n}$ with the Hilbert space

$$
L_{2}(\Omega)=\{\phi \mid(\phi, \phi)<\infty\},(\phi, \psi)=\int_{\Omega} \phi \psi d \Omega
$$

and the Sobolev space

$$
H_{0}^{1}(\Omega)=\left\{\phi \mid \phi \in L_{2}(\Omega), D^{1} \phi \in L_{2}(\Omega), \phi=0 \text { on } \partial \Omega\right\},
$$


where $D^{1}$ denotes any derivative of the first order.

For existence and uniqueness of the classical solution see [10]. For the numerical solution by finite element methods see [9].

2.2. The Crouzeix-Velte decomposition. For an $n>2$, let $(\cdot, \cdot)$ denote the Euclidean scalar product in $\mathbb{R}^{n}$, moreover, let $A, B, C \in \mathbb{R}^{n \times n}$ be matrices satisfying

$$
\begin{aligned}
A & =B+C, \\
A & =A^{T}>0, \\
B=B^{T} \geq 0 & , \quad C=C^{T} \geq 0, \\
\delta:=\operatorname{dim} \operatorname{ker} B \geq 1 & , \quad \varrho:=\operatorname{dim} \operatorname{ker} C \geq 1 .
\end{aligned}
$$

Then, with a suitable subspace $W \subset \mathbb{R}^{n}$ (which may turn out to be empty) and with orthogonality to be understood in the sense of the scalar product $(A \cdot, \cdot)$, the following orthogonal decomposition of $\mathbb{R}^{n}$ can be derived (see [15]):

$$
\mathbb{R}^{n}=\operatorname{ker} B \oplus \operatorname{ker} C \oplus W .
$$

We call this decomposition the algebraic Crouzeix-Velte decomposition of $\mathbb{R}^{n}$. It is of interest due to the following correspondences connected with the matrices in (2.5):

$$
A \sim-\Delta, B \sim-\text { grad div, } C \sim \operatorname{curl} \text { rot, }
$$

where $\Delta$ is the (vector) Laplace operator and where the sign $\sim$ expresses only an analogy between a differential operator and a matrix, and is not necessarily a (good) approximation. In this sense (2.5) corresponds to the well-known identity

$$
-\Delta=-\operatorname{grad} \operatorname{div}+\operatorname{curl} \text { rot }
$$

of vector analysis.

In the discrete case, the velocity space (which approximates $\left(H^{1}(\Omega)\right)^{d}$ or a subspace of the latter) will be denoted by $V_{h}$, the pressure space will be denoted by $P_{h}$, and $\operatorname{div}_{h}$ and $\operatorname{rot}_{h}$ will be written for the discrete equivalents of the divergence and rotation operator, $\Delta_{h}$ will denote the discrete Laplace operator. The matrix corresponding to the mapping $-\operatorname{div}_{h}$ from the velocity space into the pressure space is denoted by $\tilde{B}_{h}$ and we introduce the following notations: $\tilde{C}_{h}$ for the matrix of the operator $\operatorname{rot}_{h}$ and $A_{h}$ for the matrix of the operator $-\Delta_{h}$. If $A_{h}, \tilde{B}_{h}, \tilde{C}_{h}$ matrices satisfy the following:

$$
\begin{aligned}
& A_{h}=B_{h}+C_{h}, \\
& A_{h}=A_{h}^{T}>0,
\end{aligned}
$$

where $B_{h}=\tilde{B}_{h}^{T} \tilde{B}_{h}$ and $C_{h}=\tilde{C}_{h}^{T} \tilde{C}_{h}$ and $\operatorname{ker} \tilde{B}_{h} \neq \varnothing$ and $\operatorname{ker} \tilde{C}_{h} \neq \varnothing$, then a discrete Crouzeix-Velte decomposition exists and (2.9) takes the form

$$
V_{h}=\operatorname{ker}_{\operatorname{div}_{h}} \oplus \operatorname{ker} \operatorname{rot}_{h} \oplus W=V_{h, 0} \oplus V_{h, 1} \oplus V_{h, \beta} .
$$


$P_{h}$ is decomposed similarly into three orthogonal subspaces:

$$
P_{h}=\operatorname{kergrad}_{h} \oplus \operatorname{div}_{h} \operatorname{ker} \operatorname{rot}_{h} \oplus \operatorname{div}_{h} V_{h, \beta}=P_{h, 0} \oplus P_{h, 1} \oplus P_{h, \beta} .
$$

After discretization by the finite element or finite difference methods, the Stokes problem takes the following form ([9],[16]):

$$
\left(\begin{array}{cc}
A_{h} & \tilde{B}_{h}^{T} \\
\tilde{B}_{h} & 0
\end{array}\right)\left(\begin{array}{c}
u \\
p
\end{array}\right)=\left(\begin{array}{c}
f \\
0
\end{array}\right)
$$

For the specific formulae of these matrices in a polar coordinate system see below Section 3. With thesse matrices the Schur complement is $S_{h}:=\tilde{B}_{h} A_{h}^{-1} \tilde{B}_{h}^{T}$. For the numerical solution of the discretized Stokes problem, the eigenvalue problem for $S_{h}$ is important:

$$
S_{h} p_{h}=\lambda_{h} p_{h}
$$

If the discrete Crouzeix-Velte decomposition exists, then $\lambda_{h} \in[0,1]$ ([12]).

The analytical case of the Crouzeix-Velte decomposition for two-dimensional domains was investigated, and for the circle domain, the spectrum of the Schur complement was proved to be $0,1 / 2,1$, where $1 / 2$ and 1 have infinite multiplicity, in [3], [8] and [4]. For the analytical case a three-dimensional ball is described in [18]. The results of the spectrum for tube domains are contained in [7].

\section{The STAGGERED GRID APPROXIMATION ON A UNIT DISK}

The difference approximation on a staggered grid will be considered here. In this case, where $\Omega$ is the unit circle subdivided by an equidistant grid according to $r$ and $\varphi$ into $\mathrm{n} * \mathrm{~m}$ cells. The cell midpoints are pressure nodes, the pressure vector is denoted by $p_{h}$ and its components by $p_{i j}$, with $i=1, \ldots, n ; j=1, \ldots, m$. The sides of the cells contain as their midpoints the velocity nodes: nodes of the $u$-components of the velocity are on the sides according to $r$, nodes of the $v$-components are on the sides according to $\varphi$. The velocity vector is $\vec{u}_{h}$, its components are denoted by $u_{h}$ and $v_{h}$ and theirs components by $u_{i j}$ and $v_{i j}, i=1, \ldots, n, j=1, \ldots, m$. The boundary values are $u_{n, j}:=0$ where $j=1, \ldots, m$, further $u_{h}$ and $v_{h}$ are periodical with respect to $\varphi$, that is

$$
u_{i, 0}=u_{i, m}, \quad v_{i, 0}=v_{i, m}, \quad i=1, \ldots, n .
$$

For the approximation of the Stokes problem we need the discrete gradient, the discrete divergence operator and the vector Laplace operator. Moreover, we will also define the discrete rotation and curl operators. For the gradient:

$$
(\operatorname{grad} p)=\left(\begin{array}{c}
\frac{\partial p}{\partial r} \\
\frac{1}{r} \frac{\partial p}{\partial \varphi}
\end{array}\right)
$$


we shall use the approximation:

$$
\left(\operatorname{grad}_{h} p_{h}\right)_{i j}:=\left(\begin{array}{c}
\left(p_{i+1, j}-p_{i, j}\right) / h_{r} \\
\frac{1}{\overline{\bar{r}}_{i}}\left(p_{i, j+1}-p_{i, j}\right) / h_{\varphi}
\end{array}\right)
$$

where $h_{r}:=1 / n, h_{\varphi}:=1 / m$ and $\bar{r}_{i}:=(i-1 / 2) h_{r}, i=1, \ldots, n, j=1, \ldots, m$. For the approximation of the divergence operator, we shall use:

$$
\left(\operatorname{div}_{h} \vec{u}_{h}\right)_{i j}:=\frac{r_{i} u_{i, j}-r_{i-1} u_{i-1, j}}{\bar{r}_{i} h_{r}}+\frac{v_{i, j}-v_{i, j-1}}{\bar{r}_{i} h_{\varphi}},
$$

where $r_{i}:=i h_{r}, i=1, \ldots, n, j=1, \ldots, m$ and $r_{0} u_{0, j}=0$.

For the rotation:

$$
(\operatorname{rot} \vec{u})=\frac{1}{r}\left(\frac{\partial}{\partial r}(r v)-\frac{\partial u}{\partial \varphi}\right)
$$

we shall use the approximation:

$$
\left(\operatorname{rot}_{h} \vec{u}_{h}\right)_{i j}:=\frac{\bar{r}_{i+1} v_{i+1, j}-\bar{r}_{i} v_{i, j}}{r_{i} h_{r}}-\frac{u_{i, j+1}-u_{i, j}}{r_{i} h_{\varphi}}
$$

where $i=1, \ldots, n-1, j=1, \ldots, m$, and

$$
\left(\operatorname{rot}_{h} \vec{u}_{h}\right)_{n j}:=\frac{\bar{r}_{n} v_{n, j}}{r_{n} h_{r} / 2}
$$

where $j=1, \ldots, m$. For scalar functions, we define the operator curl as follows:

$$
(\operatorname{curl} \psi):=\left(\begin{array}{c}
\frac{1}{r} \frac{\partial \psi}{\partial \varphi} \\
-\frac{\partial \psi}{\partial r}
\end{array}\right)
$$

and we shall approximate this operator in the following way:

$$
(\operatorname{curl} \psi)_{i j}:=\left(\begin{array}{c}
\frac{1}{r_{i}} \frac{\psi_{i j}-\psi_{i, j-1}}{h_{\varphi}} \\
-\frac{\psi_{i j}-\psi_{i-1, j}}{h_{r}}
\end{array}\right) \text {. }
$$

Since

$$
(\operatorname{grad} \operatorname{div} \vec{u}-\operatorname{curl} \operatorname{rot} \vec{u})=\left(\begin{array}{l}
\frac{\partial}{\partial r}\left(\frac{1}{r} \frac{\partial}{\partial r}(r u)+\frac{1}{r} \frac{\partial v}{\partial \varphi}\right)-\frac{1}{r} \frac{\partial}{\partial \varphi}\left(\frac{1}{r} \frac{\partial}{\partial r}(r v)-\frac{1}{r} \frac{\partial u}{\partial \varphi}\right) \\
\frac{1}{r} \frac{\partial}{\partial \varphi}\left(\frac{1}{r} \frac{\partial}{\partial r}(r u)+\frac{1}{r} \frac{\partial v}{\partial \varphi}\right)+\frac{\partial}{\partial r}\left(\frac{1}{r} \frac{\partial}{\partial r}(r v)-\frac{1}{r} \frac{\partial u}{\partial \varphi}\right)
\end{array}\right),
$$

that is

$$
(\operatorname{grad} \operatorname{div} \vec{u}-\operatorname{curl} \operatorname{rot} \vec{u})=\left(\begin{array}{l}
\frac{1}{r} \frac{\partial}{\partial r}\left(r \frac{\partial u}{\partial r}\right)-\frac{u}{r^{2}}-\frac{2}{r^{2}} \frac{\partial v}{\partial \varphi}+\frac{1}{r^{2}} \frac{\partial^{2} u}{\partial \varphi^{2}} \\
\frac{1}{r} \frac{\partial}{\partial r}\left(r \frac{\partial v}{\partial r}\right)-\frac{v}{r^{2}}+\frac{2}{r^{2}} \frac{\partial u}{\partial \varphi}+\frac{1}{r^{2}} \frac{\partial^{2} v}{\partial \varphi^{2}}
\end{array}\right),
$$

identity (2.10) holds in polar coordinate system in the following form:

$$
\left(\begin{array}{l}
\Delta_{r \varphi} u-\frac{u}{r^{2}}-\frac{2}{r^{2}} \frac{\partial v}{\partial \varphi} \\
\Delta_{r \varphi} v-\frac{v}{r^{2}}+\frac{2}{r^{2}} \frac{\partial u}{\partial \varphi}
\end{array}\right)=(\operatorname{grad} \operatorname{div} \vec{u}-\operatorname{curl} \operatorname{rot} \vec{u})
$$


Using identity (3.7) and the approximation of the divergence (3.4), rotation (3.6) and curl (3.7), we can approximate the Stokes problem (2.1), (2.2), (2.3), (2.4) in the following form:

$$
\left(\begin{array}{cc}
\tilde{A}_{h} & \tilde{B}_{h}^{T} \\
\tilde{B}_{h} & 0
\end{array}\right)\left(\begin{array}{l}
\vec{u} \\
p
\end{array}\right)=\left(\begin{array}{l}
\vec{f} \\
0
\end{array}\right) .
$$

where $\tilde{B}_{h}$ corresponds to the negative divergence operator and $-\tilde{A}_{h}$ corresponds to the left side of identity (3.7) and is the following:

$$
\begin{aligned}
-\left(\tilde{A}_{h} \vec{u}_{h}\right)_{i j}:= & \frac{1}{h_{r}}\left(\frac{1}{\bar{r}_{i+1}}\left((r u)_{r}\right)_{i+1, j}-\frac{1}{\bar{r}_{i}}\left((r u)_{r}\right)_{i, j}\right)+ \\
& +\frac{1}{h_{r}}\left(\frac{1}{\bar{r}_{i+1}}\left(v_{\varphi}\right)_{i+1, j}-\frac{1}{\bar{r}_{i}}\left(v_{\varphi}\right)_{i, j}\right)+ \\
& +\frac{1}{h_{\varphi} r_{i}^{2}}\left(\left(u_{\varphi}\right)_{i, j+1}-\left(u_{\varphi}\right)_{i, j}\right)- \\
& -\frac{1}{h_{\varphi} r_{i}^{2}}\left(\left((\bar{r} v)_{\bar{r}}\right)_{i+1, j}-\left((\bar{r} v)_{\bar{r}}\right)_{i+1, j-1}\right) \\
-\left(\tilde{A}_{h} \vec{v}_{h}\right)_{i j}:= & \frac{1}{h_{r}}\left(\frac{1}{r_{i}}\left((\bar{r} v)_{\bar{r}}\right)_{i+1, j}-\frac{1}{r_{i-1}}\left((\bar{r} v)_{\bar{r}}\right)_{i, j}\right)+ \\
& +\frac{1}{h_{r}}\left(\frac{1}{r_{i-1}}\left(u_{\varphi}\right)_{i-1, j+1}-\frac{1}{r_{i}}\left(u_{\varphi}\right)_{i, j+1}\right)+ \\
& +\frac{1}{h_{\varphi} \bar{r}_{i}^{2}}\left(\left(v_{\varphi}\right)_{i, j+1}-\left(v_{\varphi}\right)_{i, j}\right)- \\
& -\frac{1}{h_{\varphi} \bar{r}_{i}^{2}}\left(\left((r u)_{r}\right)_{i, j+1}-\left((r u)_{r}\right)_{i, j}\right),
\end{aligned}
$$

where $i=1, \ldots, n, j=1, \ldots, m$ and taking into account (3.1), and we used the following notations:

$$
\begin{aligned}
\left(u_{\varphi}\right)_{i, j}: & :=\frac{u_{i, j}-u_{i, j-1}}{h_{\varphi}}, \\
\left(v_{\varphi}\right)_{i, j}: & :=\frac{v_{i, j}-v_{i, j-1}}{h_{\varphi}}, \\
\left((r u)_{r}\right)_{i, j}: & :=\frac{r_{i} u_{i, j}-r_{i-1} u_{i-1, j}}{h_{r}}, \\
\left((\bar{r} v)_{\bar{r}}\right)_{i, j} \quad: & =\frac{\bar{r}_{i} v_{i, j}-\bar{r}_{i-1} v_{i-1, j}}{h_{r}} .
\end{aligned}
$$


We also introduce the following notation: $\tilde{C}_{h}$ for the matrix of the operator $\operatorname{rot}_{h}$.

For pressure vectors $p_{h}, q_{h}$ and velocity vectors $\vec{u}_{h}=\left(u_{h}, v_{h}\right)^{T}, \quad \vec{w}_{h}=\left(t_{h}, s_{h}\right)^{T}$ the following discrete scalar products and the corresponding norms are introduced: and

$$
\begin{aligned}
&\left(p_{h}, q_{h}\right)_{0, r, h}:= \sum_{i=1}^{n} \sum_{j=1}^{m} p_{i j} q_{i j} r_{i} h_{r} h_{\varphi}, \\
&\left\|p_{h}\right\|_{0, r, h}^{2}:=\quad\left(p_{h}, p_{h}\right)_{0, r, h},
\end{aligned}
$$

and

$$
\begin{aligned}
\left(\vec{u}_{h}, \vec{w}_{h}\right)_{0, h}:= & \sum_{i=1}^{n} \sum_{j=1}^{m}\left(u_{i j} t_{i j} r_{i}+v_{i j} s_{i j} \bar{r}_{i}\right) h_{r} h_{\varphi} \\
\left\|\vec{u}_{h}\right\|_{0, h}^{2}:= & \left(\vec{u}_{h}, \vec{u}_{h}\right)_{0, h} .
\end{aligned}
$$

Theorem 1. $\left(\tilde{A}_{h} \vec{u}_{h}, \vec{u}_{h}\right)_{0, h}=\left\|\tilde{B}_{h} \vec{u}_{h}\right\|_{0, \bar{r}, h}^{2}+\left\|\tilde{C}_{h} \vec{u}_{h}\right\|_{0, r, h}^{2} \quad$ holds for all vectors $\vec{u}_{h}:=$ $\left(u_{h}, v_{h}\right)^{T} \in \vec{V}_{h}$ 
Proof. We apply partial summation to $\left(\tilde{A}_{h} \vec{u}_{h}, \vec{u}_{h}\right)_{0, h}$ :

$$
\begin{aligned}
\left(\tilde{A}_{h} \vec{u}_{h}, \vec{u}_{h}\right)_{0, h} & =\sum_{i=1}^{n-1} \sum_{j=1}^{m} \frac{h_{\varphi}}{\bar{r}_{i+1} h_{r}}\left(r_{i+1} u_{i+1, j}-r_{i} u_{i, j}\right)^{2}+ \\
& +\sum_{j=1}^{m} \frac{h_{\varphi}}{\bar{r}_{1} h_{r}}\left(r_{1} u_{1, j}\right)^{2}+\sum_{i=1}^{n} \sum_{j=1}^{m} \frac{h_{r}}{r_{i} h_{\varphi}}\left(u_{i, j+1}-u_{i, j}\right)^{2}- \\
& -\sum_{i=1}^{n} \sum_{j=1}^{m}\left(\frac{r_{i}}{\bar{r}_{i+1}}\left(v_{i+1, j} u_{i, j}-v_{i+1, j-1} u_{i, j}\right)-\frac{r_{i}}{\bar{r}_{i}}\left(v_{i, j} u_{i, j}-v_{i, j-1} u_{i, j}\right)\right)- \\
& -\sum_{i=1}^{n} \sum_{j=1}^{m}\left(\frac{\bar{r}_{i+1}}{r_{i}}\left(v_{i+1, j-1} u_{i, j}-v_{i+1, j} u_{i, j}\right)-\frac{\bar{r}_{i}}{r_{i}}\left(v_{i, j-1} u_{i, j}-v_{i, j} u_{i, j}\right)\right)+ \\
& +\sum_{i=1}^{n-1} \sum_{j=1}^{m} \frac{h_{\varphi}}{r_{i} h_{r}}\left(\bar{r}_{i+1} v_{i+1, j}-\bar{r}_{i} v_{i, j}\right)^{2}+ \\
& +\sum_{j=1}^{m} \frac{h_{\varphi}}{r_{n} h_{r}}\left(\bar{r}_{n} v_{n, j}\right)^{2}+\sum_{i=1}^{n} \sum_{j=1}^{m} \frac{h_{r}}{\bar{r}_{i} h_{\varphi}}\left(v_{i, j+1}-v_{i, j}\right)^{2}- \\
& -\sum_{i=1}^{n} \sum_{j=1}^{m}\left(\frac{\bar{r}_{i}}{r_{i-1}}\left(u_{i-1, j+1} v_{i, j}-u_{i-1, j} v_{i, j}\right)-\frac{\bar{r}_{i}}{r_{i}}\left(u_{i, j+1} v_{i, j}-u_{i, j} v_{i, j}\right)\right)- \\
& -\sum_{i=1}^{n} \sum_{j=1}^{m}\left(\frac{r_{i-1}}{\bar{r}_{i}}\left(u_{i-1, j} v_{i, j}-u_{i-1, j+1} v_{i, j}\right)-\frac{r_{i}}{\bar{r}_{i}}\left(u_{i, j} v_{i, j}-u_{i, j+1} v_{i, j}\right)\right) .
\end{aligned}
$$

$\left\|\tilde{B}_{h} \vec{u}_{h}\right\|_{0, \bar{r}, h}^{2}$ may be written in the following form:

$$
\begin{aligned}
\left\|\tilde{B}_{h} \vec{u}_{h}\right\|_{0, \bar{r}, h}^{2} & =\sum_{i=1}^{n} \sum_{j=1}^{m}\left(\frac{h_{\varphi}}{\bar{r}_{i} h_{r}}\left(r_{i} u_{i, j}-r_{i-1} u_{i-1, j}\right)^{2}+\right. \\
& \left.+\frac{h_{r}}{\bar{r}_{i} h_{\varphi}}\left(v_{i, j}-v_{i, j-1}\right)^{2}+\frac{2}{\bar{r}_{i}}\left(r_{i} u_{i, j}-r_{i-1} u_{i-1, j}\right)\left(v_{i, j}-v_{i, j-1}\right)\right) .
\end{aligned}
$$

$\left\|\tilde{C}_{h} \vec{u}_{h}\right\|_{0, r, h}^{2}$ can be written as follows:

$$
\begin{aligned}
\left\|\tilde{C}_{h} \vec{u}_{h}\right\|_{0, r, h}^{2} & =\sum_{i=1}^{n} \sum_{j=1}^{m}\left(\frac{h_{r}}{r_{i} h_{\varphi}}\left(u_{i, j+1}-u_{i, j}\right)^{2}+\frac{h_{\varphi}}{r_{i} h_{r}}\left(\bar{r}_{i+1} v_{i+1, j}-\bar{r}_{i} v_{i, j}\right)^{2}-\right. \\
& \left.-\frac{2}{r_{i}}\left(u_{i, j+1}-u_{i, j}\right)\left(\bar{r}_{i+1} v_{i+1, j}-\bar{r}_{i} v_{i, j}\right)\right) .
\end{aligned}
$$


Performing some index shifts and taking into account the periodicity in the direction $\varphi$ and the homogeneous boundary conditions we get:

$$
\left(\tilde{A}_{h} \vec{u}_{h}, \vec{u}_{h}\right)_{0, h}-\left\|\tilde{B}_{h} \vec{u}_{h}\right\|_{0, \bar{r}, h}^{2}-\left\|\tilde{C}_{h} \vec{u}_{h}\right\|_{0, r, h}^{2}=0 .
$$

Remark 1. $\tilde{A}_{h}$ a symmetric matrix in the sense of the scalar product (3.13). This means that Theorem can be described in matrix terms as follows:

$$
\begin{aligned}
\left(D_{\tilde{A}} \tilde{A}_{h} \vec{u}_{h}, \vec{u}_{h}\right) & =\left(D_{\tilde{B}} \tilde{B}_{h} \vec{u}_{h}, \tilde{B}_{h} \vec{u}_{h}\right)+\left(D_{\tilde{C}} \tilde{C}_{h} \vec{u}_{h}, \tilde{C}_{h} \vec{u}_{h}\right)= \\
& =\left(\tilde{B}_{h}^{T} D_{\tilde{B}} \tilde{B}_{h} \vec{u}_{h}, \vec{u}_{h}\right)+\left(\tilde{C}_{h}^{T} D_{\tilde{C}} \tilde{C}_{h} \vec{u}_{h}, \vec{u}_{h}\right),
\end{aligned}
$$

where (...) is the Euclidean scalar product and $D_{\tilde{A}}, D_{\tilde{B}}, D_{\tilde{C}}$ are diagonal matrices corresponding to (3.13) and (3.12):

$$
\begin{aligned}
& D_{\tilde{A}}=\operatorname{diag}\left(r_{1} I_{m \times m}, \ldots, r_{n-1} I_{m \times m}, \bar{r}_{1} I_{m \times m}, \ldots, \bar{r}_{n} I_{m \times m}\right), \\
& D_{\tilde{B}}=\operatorname{diag}\left(\bar{r}_{1} I_{m \times m}, \ldots, \bar{r}_{n} I_{m \times m},\right), \\
& D_{\tilde{C}}=\operatorname{diag}\left(r_{1} I_{m \times m}, \ldots, r_{n} I_{m \times m},\right) .
\end{aligned}
$$

From (3.17) we get:

$$
D_{\tilde{A}} \tilde{A}_{h}=\tilde{B}_{h}^{T} D_{\tilde{B}} \tilde{B}_{h}+\tilde{C}_{h}^{T} D_{\tilde{C}} \tilde{C}_{h}
$$

That is $D_{\tilde{A}} \tilde{A}_{h}$ is a symmetric matrix and can be written in the following form:

$$
D_{\tilde{A}} \tilde{A}_{h}=: A_{h}=B_{h}+C_{h},
$$

where $B_{h}=\hat{B}_{h}^{T} \hat{B}_{h}$ and $C_{h}=\hat{C}_{h}^{T} \hat{C}_{h}$ with the notation $\hat{B}_{h}=D_{\tilde{B}}^{1 / 2} \tilde{B}_{h}, \hat{C}_{h}=D_{\tilde{C}}^{1 / 2} \tilde{C}_{h}$.

\section{Numerical Results}

Using the notations (3.20), our problem consists in finding the solution of the following algebraic system:

$$
\begin{aligned}
A_{h} \vec{u}_{h}+\hat{B}_{h}^{T} p_{h} & =\vec{f}_{h}, \\
\hat{B}_{h} \vec{u}_{h} & =g_{h} .
\end{aligned}
$$

We use the Uzawa-algorithm ([9]) to solve (4.1), (4.2):

$$
\begin{aligned}
p_{h}^{(0)} & :=0, \\
p_{h}^{(i+1)} & :=p_{h}^{(i)}+\omega\left(\hat{B}_{h} \vec{u}_{h}^{(i)}-g_{h}\right) \\
\vec{u}_{h}^{(i)} & :=A_{h}^{-1}\left(\vec{f}_{h}-\hat{B}_{h}^{T} p_{h}^{(i)}\right) \\
i=0,1,2, \ldots &
\end{aligned}
$$


(4.3) can be written in the following form:

$$
\begin{aligned}
p_{h}^{(0)} & =0 \\
p_{h}^{(i+1)} & =p_{h}^{(i)}+\omega\left(\psi_{h}-S_{h} p_{h}^{(i)}\right) \\
i=0,1,2, \ldots &
\end{aligned}
$$

where $\psi_{h}=\hat{B}_{h} A_{h}^{-1} \vec{f}_{h}-g_{h}$ and $S_{h}$ is the discrete Schur complement operator, that is $S_{h}=\hat{B}_{h} A_{h}^{-1} \hat{B}_{h}^{T}$. Since the discrete Crouzeix-Velte decomposition exists, using the Uzawa-algorithm we can reach the third Crouzeix-Velte subspace after at most 2 steps ([13]). In this subspace the spectrum of the Schur complement is closer, and the algorithm performs effective convergence. We introduce the following notations: the optimal iteration parameter is $\omega_{o p t}^{h}:=2 /\left(\underline{\lambda}_{h}+\bar{\lambda}_{h}\right)$, where $\underline{\lambda}_{h}$ and $\bar{\lambda}_{h}$ are the smallest and the largest of the eigenvalues different from 0 and 1 of the discrete Schur complement; and $\omega=2$ is the optimal iteration parameter for the undiscretized Uzawaalgorithm, see ([13]). Table 1 shows the values of the optimal iteration parameter $\left(\omega_{\text {opt }}^{h}\right)$ and the smallest and largest eigenvalues $\left(\underline{\lambda}_{h}, \bar{\lambda}_{h}\right)$.

Table 1

\begin{tabular}{|l|ccccc|}
\hline$n=m$ & 5 & 10 & 20 & 40 & 60 \\
\hline$\underline{\lambda}_{h}$ & 0.5133234 & 0.5036472 & 0.5009311 & 0.5002340 & 0.5001041 \\
\hline $\bar{\lambda}_{h}$ & 0.5248821 & 0.5188427 & 0.5154488 & 0.5138629 & 0.5133509 \\
\hline$\omega_{o p t}^{h}$ & 1.926401 & 1.95601 & 1.967768 & 1.972198 & 1.973447 \\
\hline
\end{tabular}

In a first numerical experiment in (4.1), (4.2) we took random values for the exact solution of $p_{h}$ which was projected to the orthogonal complement of the kernel of $\hat{B}_{h}^{T}$. Here $\psi_{h}$ was calculated as $\psi_{h}:=S_{h} p_{h}$. After $i=1,2$ initial steps with $\omega=$ 1 , the optimal parameters have been taken. In Table $2\left\langle i ; \omega_{\text {opt }}^{h}\right\rangle$ means the number of iterations (included the initial steps) in the case of $i$ initial steps and after that iterations with $\omega_{o p t}^{h}$. $\langle i ; \omega=2\rangle$ means the number of iterations in the case of $i$ initial steps followed by iterations with $\omega=2$. For comparison we show the number of iterations in the case of $\omega=1$ iteration parameter in all steps, which is in widespread use, denoted by $\langle 1 ; \omega=1\rangle$. The numerical convergence rate $q:=\left(\left\|e^{(i t)}\right\| /\left\|e^{(0)}\right\|\right)^{1 / i t}$ is also shown, where $e^{(0)}$ is the initial pressure error and $e^{(i t)}$ is the final error after it iterations. The stopping criterion is that the initial pressure error in the Euclidean norm has been reduced by a factor of $10^{-5}$. In the inner iteration (in the conjugate gradient method for details see below), it is necessary to use a stronger stopping criterion: that the initial error in the Euclidean norm has been decreased at least by a factor of $10^{-6}$. (If in the outer iteration the initial pressure error is reduced by a factor of $10^{-10}$, then in the inner iteration it is necessary to decrease the initial error at least by a factor of $10^{-11}$.) 
Table 2

\begin{tabular}{|c|ccccc|}
\hline$n=m$ & 5 & 10 & 20 & 40 & 60 \\
\hline$\langle 1 ; \omega=1\rangle$ & 14 & 15 & 16 & 17 & 17 \\
\hline$q$ & 0.4179 & 0.4172 & 0.4131 & 0.4166 & 0.4129 \\
\hline$\left\langle 1 ; \omega_{\text {opt }}^{h}\right\rangle$ & 4 & 4 & 4 & 4 & 4 \\
\hline$q$ & 0.0056 & 0.0057 & 0.0046 & 0.0041 & 0.0041 \\
\hline$\langle 1 ; \omega=2\rangle$ & 5 & 4 & 4 & 4 & 4 \\
\hline$q$ & 0.0291 & 0.0138 & 0.0105 & 0.0090 & 0.0083 \\
\hline$\left\langle 2 ; \omega_{\text {opt }}^{h}\right\rangle$ & 4 & 5 & 5 & 5 & 5 \\
\hline$q$ & 0.0029 & 0.0044 & 0.0041 & 0.0034 & 0.0032 \\
\hline$\langle 2 ; \omega=2\rangle$ & 5 & 5 & 5 & 5 & 5 \\
\hline$q$ & 0.0144 & 0.0106 & 0.0090 & 0.0074 & 0.0067 \\
\hline
\end{tabular}

In the second numerical experiment, an algebraic Stokes problem with known solution was generated. (The exact solution is: $u_{\text {exact }}=r \cos \varphi+2 r \sin \varphi+3$, $v_{\text {exact }}=4 r \cos \varphi-r \sin \varphi+2$ and $p_{\text {exact }}=2 r \cos \varphi$. In this case $\operatorname{div}(u, v)=0$ and the solvability condition of (4.1), (4.2) is satisfied.) The number of outer iterations and the numerical convergence rate are shown in Table 3 depending on the iteration parameter. Here, iteration was stopped if the initial pressure error had been reduced by a factor of $10^{-6}$. In the inner iteration the initial error in the Euclidean norm was decreased by a factor of $10^{-7}$.

Table 3

\begin{tabular}{|c|cccccc|}
\hline$n=m$ & 5 & 10 & 20 & 40 & 60 & 80 \\
\hline$\langle 1 ; \omega=1\rangle$ & 21 & 22 & 23 & 24 & 25 & 25 \\
\hline$q$ & 0.4693 & 0.5022 & 0.5606 & 0.5784 & 0.6354 & 0.6143 \\
\hline$\left\langle 1 ; \omega_{\text {opt }}^{h}\right\rangle$ & 5 & 5 & 5 & 5 & 5 & - \\
\hline$q$ & 0.0341 & 0.0690 & 0.0742 & 0.0932 & 0.0110 & - \\
\hline$\langle 1 ; \omega=2\rangle$ & 5 & 4 & 4 & 4 & 3 & 3 \\
\hline$q$ & 0.0342 & 0.0283 & 0.0312 & 0.0422 & 0.0043 & 0.00731 \\
\hline$\left\langle 2 ; \omega_{\text {opt }}^{h}\right\rangle$ & 5 & 6 & 6 & 6 & 6 & - \\
\hline$q$ & 0.0112 & 0.0690 & 0.0742 & 0.0932 & 0.0092 & - \\
\hline$\langle 2 ; \omega=2\rangle$ & 6 & 5 & 5 & 4 & 4 & 4 \\
\hline$q$ & 0.0341 & 0.0283 & 0.0312 & 0.0087 & 0.0043 & 0.0073 \\
\hline
\end{tabular}

Because of the large amount of memory needed, $\omega_{o p t}^{h}$ was not calculated in the case of $n=m=80$.

Instead of the calculation of $A_{h}^{-1}$ in (4.3) we used the fast Fourier transformation in combination with the preconditioned conjugate gradient method. For this, let us introduce the following notations:

$$
\begin{aligned}
\vec{u}_{h, F} & :=Q_{2 n-1}^{*} \vec{u}_{h}, & p_{h, F}:=Q_{n}^{*} p_{h}, \\
A_{h, F} & :=Q_{2 n-1}^{*} A_{h} Q_{2 n-1}, & \hat{B}_{h, F}:=Q_{n}^{*} \hat{B}_{h} Q_{2 n-1},
\end{aligned}
$$


where $Q_{k}$, the matrix of the Fourier transformation, is a block-diagonal matrix with $k$ blocks $Q=\left(q_{j, l}\right)_{j, l=1}^{m}$ and $q_{j, l}=\sqrt{1 / m} e^{i h_{\varphi} l j}$. Now, instead of (4.3) the following iteration for the transformed variables can be used:

$$
\begin{aligned}
\vec{f}_{h, F} & :=Q_{2 n-1}^{*} \vec{f}_{h}, \quad g_{h, F}:=Q_{n}^{*} g_{h}, \\
p_{h, F}^{(0)} & :=0, \\
i=0,1,2, \ldots, \max _{i t}: & \\
{\left[p_{h, F}^{(i+1)}\right.} & :=p_{h, F}^{(i)}+\omega\left(\hat{B}_{h, F} \vec{u}_{h, F}^{(i)}-g_{h, F}\right) \\
\vec{u}_{h, F}^{(i)} & \left.:=A_{h, F}^{-1}\left(\vec{f}_{h, F}-\hat{B}_{h, F}^{*} p_{h, F}^{(i)}\right)\right] \\
\vec{u}_{h} & :=Q_{2 n-1} \vec{u}_{h, F}, \quad p_{h}:=Q_{n} p_{h, F},
\end{aligned}
$$

Observe that in a cartesian coordinate system, $A_{h, F}$ would be block-diagonal with tridiagonal blocks. In our case of polar coordinates, however, $A_{h, F}$ has 7 nonzero diagonals, the main diagonal, the $\pm m$-th, the $\pm(m-1) * n$-th and the $\pm(m-1) *$ $n+m$-th diagonal (using the Matlab notation). To solve the equation $A_{h, F} x=b$ the preconditioned conjugate gradient method was used combined with incomplete Gauss-elimination. (Additional diagonals were not used in the incomplete Gausselimination. This version is sometimes called ILU(0).) In the numerical experiments several ILU(0)-type preconditioning matrices fitting the structure of $A_{h, F}$ were investigated and $T_{o p t}$ was found to be the optimal preconditioner from the 5 preconditioners considered, resulting in the fastest convergence with a few number of iterations. $T_{o p t}$ is a tridiagonal matrix consisting of the main diagonal and the $m$-th and $-m$-th diagonal of $A_{h, F}$. In the numerical experiments above, this preconditioning matrix was used. Table 4 displays the number of inner iterations needed to reach the stopping criterion of the conjugate gradient method, in that the initial error in the Euclidean norm has been decreased by a factor of $10^{-4}$. In the Table $T_{\text {opt }}$ shows that the matrix $T_{\text {opt }}$ was used as the preconditioning matrix, and the unpreconditioned conjugate gradient method is denoted by $T=I$. For comparison we show the number of iterations with the preconditioning matrix, denoted by $T_{D}$, which contains only the main diagonal of $A_{h, F}$ and two other preconditioning matrices denoted by $T_{*}$ and $T_{* *}$. Here $T_{*}$ is a tridiagonal matrix consisting of the main diagonal and the $(m-1) * n+m$-th and $-((m-1) * n+m)$-th diagonal of $A_{h, F}$ and $T_{* *}$ is a pentadiagonal matrix with the main diagonal and the $\pm m$-th and $\pm(m-1) * n+m$-th diagonal of $A_{h, F}$.

Table 4

\begin{tabular}{|l|cccccccc|}
\hline$n=m$ & 5 & 10 & 20 & 50 & 100 & 200 & 500 & 640 \\
\hline$T_{\text {opt }}$ & 9 & 12 & 12 & 12 & 8 & 5 & 5 & 4 \\
\hline$T=I$ & 25 & 59 & 76 & 197 & 362 & - & - & - \\
\hline$T_{D}$ & 19 & 39 & 72 & 106 & 125 & - & - & - \\
\hline$T_{*}$ & 19 & 39 & 72 & 107 & 125 & - & - & - \\
\hline$T_{* *}$ & 7 & 8 & 8 & 8 & 5 & 3 & 2 & 2 \\
\hline
\end{tabular}


Because of the large amount of computational time needed, the number of the iteration was not calculated in the case of $n=m \geq 200$, and the preconditioning matrices are $T=I, T_{D}$ or $T_{*}$.

In Table 5 we show the computational time of the conjugate gradient method (inner iteration) to reach our stopping criterion $10^{-4}$, and the necessary memory for the incomplete Gauss-elimination using the different preconditioning matrices and $n=$ $m=50$.

Table 5

\begin{tabular}{|l|cc|}
\hline$n=m=50$ & computational time (s) & memory needed (byte) \\
\hline$T_{o p t}$ & 32.28 & 2400 \\
\hline$T=I$ & 202.70 & - \\
\hline$T_{D}$ & 163.95 & 800 \\
\hline$T_{*}$ & 326.47 & 2400 \\
\hline$T_{* *}$ & 63.92 & 4000 \\
\hline
\end{tabular}

The full computational time of the second numerical experiment in the case of $\langle 1 ; \omega=2\rangle$ and $n=m=50$ is the following: the time of the FFT and IFFT and the calculation of $\psi_{h}$ are altogether 25,155s and the full time of the outer iteration (see $(4.7), \max _{i t}=3$ ) is $121.125 \mathrm{~s}$. Here the stopping criterion of the inner iteration is $10^{-4}$ and the stopping criterion of the outer iteration is $10^{-3}$ and we used $T_{\text {opt }}$ as the preconditioning matrix.

\section{REFERENCES}

[1] BrezzI, F.: On the existence, uniqueness and approximation of saddle-point problems arising from Lagrange multipliers. R.A.I.R.O., Anal. Numer. 8 (1974), 129-151.

[2] Brezzi, F. And Fortin, M.: Hybrid and Mixed Finite Elements Methods. Springer, N.Y. 1991.

[3] Chizhonkov, E. V.: On the Constant in the LBB Condition for Ring Domains. Department of Mathematics, University of Nijmegen, The Netherlands, Report No. 9537 (1995).

[4] Crouzeix, M.: Étude d'une méthode de linéarisation. Résolution numérique des équations Stokes stationnaires. In: Cahier de l'INRIA 12, pp. 139-244 (1974).

[5] Crouzeix, M.: On an operator related to the convergence of Uzawa's algorithm for the Stokes equation. In: Computational Science for the 21st Century (Périaux, J., et al., eds. N.Y., Wiley 1997, 242-249.

[6] Dobrowolski, M., Stoyan, G.: Algebraic and discrete Velte decompositions. BIT 41, No. 3 (2001), 465-479.

[7] Dobrowolski, M.: On the LBB-Constant on Stretched Domains. Math. Nachr. 254-255 (2003) 64-67.

[8] FRIEDRICHS, K.O.: On certain inequalities and characteristic value problems for analytic functions of two variables. Trans. Amer. Math. Soc. 41, (1937), 321-364.

[9] Girault, V. and Raviart, P.-A.: Finite Element Methods for Navier-Stokes Equations. Springer, Berlin 1986.

[10] Kratz W.: The maximum modulus theorem for the Stokes system in a ball. Mathematische Zeitschrift, 226, 389-403 (1997).

[11] Ladyzhenskaya, O. A.: Mathematical Theory of Viscous Incompressible Flow. 2. ed., Gordon and Breach, New York 1969. 
[12] Stoyan, G.: Towards discrete Velte decompositions and narrow bounds for inf-sup constants. Computers \& Maths. with Appls., 38, 7-8 (1999), 243-261.

[13] Stoyan, G.: Iterative Stokes solvers in the harmonic Velte subspace. Computing 67 (2000), 13-33.

[14] Stoyan, G., Strauber, Gy. and Baran, A.: Generalizations to discrete and analytical CrouzeixVelte decompositions. (in print in Numer. Lin. Algebra).

[15] Stoyan, G.: $\Delta=-$ grad div + rot rot for matrices, with application to the finite element solution of the Stokes problem. East-West Journal of Numerical Mathematics 8, No. 4 (2000), 323-340.

[16] Strauber, Gy.: Discrete Crouzeix-Velte decompositions on nonequidistant rectangular grids. Annales Univ. Sci. Budapest.,44 (2002), 63-82.

[17] Varhorn, W.: The Stokes Equations. Akademie Verlag, Berlin, 1994.

[18] Velte, W.: On optimal constants in some inequalities. In: The Navier-Stokes Equations; Theory and Numerical Methods (J.G. Heywood et al., eds.). Lecture Notes in Math. 1431, pp. 158-168. Berlin: Springer 1990.

\section{Author's Address}

\section{Gy. Strauber:}

College of Dunaújváros, Dept. of Informatics, Táncsics M. u. 1/a, H-2400 Dunaújváros, Hungary E-mail address: strauber@mail.poliod.hu 